\title{
Modeling of a magnetically enhanced vacuum arc thruster
}

\author{
Michael Keidar ${ }^{*}$ \\ University of Michigan, Ann Arbor 48109 MI \\ Jochen Schein $^{\dagger}$ \\ Alameda Applied Science Corporation, San Leandro, CA 94577
}

The hydrodynamic model of the vacuum arc thruster and its plume is developed. Primarily an effect of the magnetic field on the plume expansion and thruster operational conditions is considered. Two particular examples are investigated namely magnetically enhanced co-axial vacuum arc thruster (MVAT) and vacuum arc thruster with ring electrodes (RVAT). It was found that magnetic field significantly decreases plasma plume expansion, while on the other hand causes arc voltage increase. Predicted plasma density profiles in the plume of the MVAT were compared with experiment and generally good agreement was found. In the case of RVAT it was found that magnetic field leads to plasma jet deceleration. This effect explains nonmonotonic dependence of the ion current density with an axial magnetic field observed experimentally.

\section{Introduction}

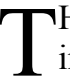
HE vacuum arc thruster (VAT) is a simple, yet efficient electric propulsion device that combined with an inductive energy storage PPU results in a low mass $(<300 \mathrm{~g})$ system. The basic mechanism underlying the VAT is the production of fully ionized micro-plasmas (cathode spots) on the cathode surface, which expand into vacuum at high velocities thus producing thrust.

The metal plasma formed by a vacuum arc discharge is created on the cathode surface at cathode spots. Optical observations indicate that the spot has a homogeneous bright region with a typical width of $10-100 \mu \mathrm{m}^{1,2,3}$. The cathodic plasma expands away from the cathode in a direction normal to the surface with a speed that is typically 10 to $30 \mathrm{~km} / \mathrm{s}$. This magnitude is as much as 10 times the speed of sound ${ }^{4}$. The pioneering work of Plyutto et al. ${ }^{5}$ and Davis and Miller ${ }^{6}$ demonstrated that more than $95 \%$ of the material that evaporates from the spot surface emerges from the spot plasma as ions. The characteristics of vacuum arc plasmas have been investigated and described by many authors ${ }^{7,8}$. An interesting feature of the vacuum arc, in contrast to gaseous plasma, is that the vacuum arc plasma is fully ionized and has a mean charge state generally greater than unity. A vacuum arc with a ring anode geometry is of interest for spacecraft propulsion applications. For this geometry, some limited measurements of the plasma parameter distribution in the cathodic plasma jet were performed. The angular ion current density distribution and the radial profile of the ion current and plasma potential were studied ${ }^{9,10}$. The measured ion distribution without a magnetic field was a slightly flattened cosinusoidal function. With an axial magnetic field, the ion distribution became peaked along the axial axis. Recently, the ion charge state distribution ${ }^{11},{ }^{12}$ and ion velocities $^{13}$ were measured using time-of-flight and current "spike" techniques. These measurements were performed for most of the conducting elements of the Periodic Table and a correlation between the element dependent ion velocity and average charge state was found. Refractory metal ions such as Mo and W have the highest energy (150-200 eV). This energy is much higher than the arc voltage equivalent (about $20 \mathrm{~V}$ ), therefore leaving open the question of the ion acceleration mechanism in the cathode spot. Theoretically it was shown that the gas-dynamic mechanism is responsible for ion acceleration ${ }^{14,15}$. More completely, the mechanisms of the plasma

\footnotetext{
${ }^{*}$ Research Fellow, Aerospace Department, 3038 FXB, AIAA Senior Member.

${ }^{\dagger}$ now with Lawrence Livermore Nat. Lab., Livermore, CA 
acceleration in the cathode spot were analyzed by Beilis et al. ${ }^{16,17}$. They concluded that the supersonic velocity of the cathode plasma jet is due to momentum transfer from the electrons to the ions by friction and an ambipolar electric field.

\section{AASC vacuum arc thruster}

The need for a low mass propulsion system motivated the development of the vacuum arc thruster. A pulsed plasma thruster that uses conductive cathode materials as propellant is combined with an energy storage power processing unit that takes $5 \mathrm{~V}-24 \mathrm{~V}$ from the bus and converts it into an adequate power pulse for the thruster. It is a system well suited to provide small impulse bits $(\approx 1 \mu \mathrm{Ns})$ at high Isp $(1000 \mathrm{~s}-3000 \mathrm{~s})$. Applications include positioning and drag makeup for small, power and mass limited satellites.

The performance of the vacuum arc thruster is determined by the propellant mass, the degree of ionization of the plasma, the angle of expansion, the average charge state and the ion velocity. All these parameters have been measured repeatedly in the past and verified for numerous materials and operating conditions. Typical values for the ion velocity vary between $10000 \mathrm{~m} / \mathrm{s}$ and $30000 \mathrm{~m} / \mathrm{s}$, the average arc/ion current ratio has been shown to be of the order $8 \%$ and a cosine distribution has been found to emulate the plasma plume expansion very well.

In order to produce a low-mass system, the vacuum arc thruster was constructed using an inductive energy storage (IES) circuit PPU and simple thruster head geometry. In the PPU, an inductor is charged through a semiconductor switch. When the switch is opened, a voltage peak Ldi/dt is produced, which breaks down the thin metal film coated anode cathode insulator surface at relatively low voltage levels $(\approx 200 \mathrm{~V})$. The current that was flowing in the solidstate switch (for $\leq 1 \mathrm{~ms}$ ) is fully switched to the vacuum arc load. Typical currents of $\sim 100 \mathrm{~A}$ (for $\sim 100-500 \mu \mathrm{s}$ ) are conducted with voltages of $\sim 25-30 \mathrm{~V}$. Consequently, most of the magnetic energy stored in the inductor is deposited into the plasma pulse. The efficiency of the PPU may thus be $>90 \%$. A picture of the VAT system is shown in figure 1. Additional information about the principle of the vacuum arc thruster can be found elsewhere. ${ }^{18,19,20}$

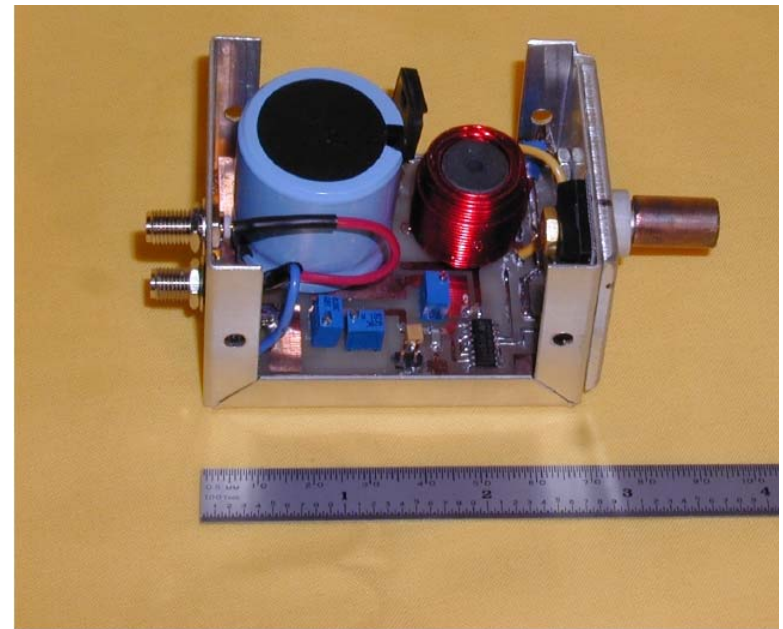

Figure 1: Vacuum Arc Thruster System including PPU (thruster head is on the right)

While the system is simple and can be realized with an extremely low mass the divergence of the plasma plume reduces the efficiency of the system due to the variations in the thrust vector and it also increases the chances of contamination of essential parts of the spacecraft. Therefore an axial magnetic field is used to control the plasma expansion. The magnetic field is produced by a coil wrapped around the anode of a coaxial thruster head (figure 2). For the purpose of performing initial experiments the currents in the coil and in the thruster are controlled independently, while a final design will utilize the magnetic field produced in the inductor of the IES PPU to control the plasma plume. ${ }^{21}$ 


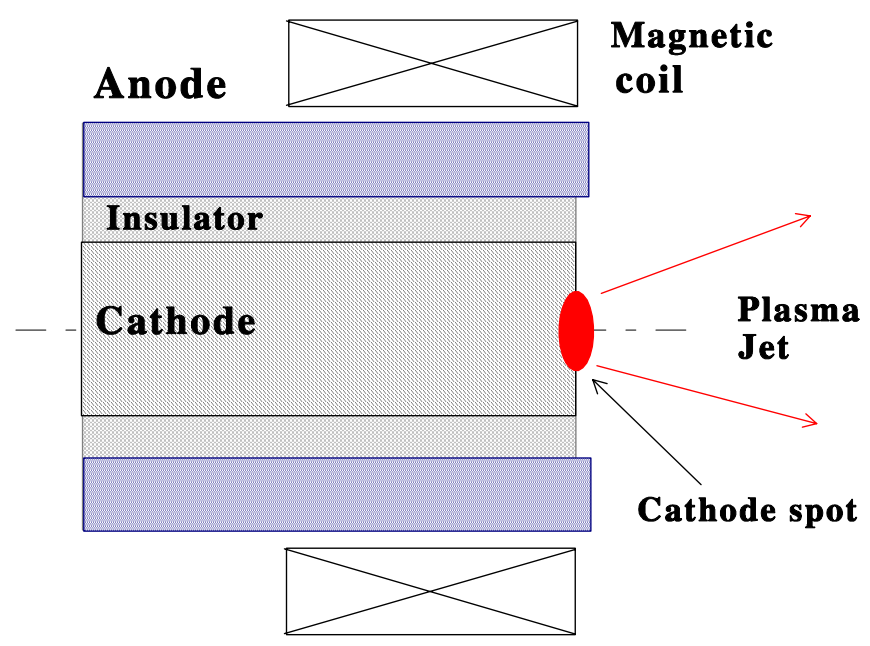

Figure 2. Schematics of the magnetically enhanced vacuum arc thruster

It has been found that by applying an axial magnetic field $(0.1 \mathrm{~T})$ around the cathode not only the plasma plume is confined but also that the ion velocities increase, leading to an improved thrust-to-power ratio. ${ }^{21}$ In order to better understand the influence of the magnetic field and to produce a highly efficient thruster design a model was developed for the magnetically enhanced vacuum arc thruster (MVAT).

\section{The model}

In the vacuum arc, the plasma jet expansion strongly depends on the processes occurring in the near cathode region in which plasma is generated. In addition, the character of the plasma expansion depends on the interelectrode gap geometry. In principle the problem of the plasma expansion in the vacuum arc has two-dimensional features. First consistent 2D analysis of the plasma flow taken into account both plasma generation effects and geometry was performed by Keidar, Beilis et. al ${ }^{22,23,24,25,26}$. In these models an effect of externally imposed magnetic field was also studied. The peculiarity of the proposed approach is in that the plasma boundary (free boundary) was treated selfconsistently. It was found that plasma jet has a conical shape and imposition of an axial magnetic field significantly alter plasma expansion. The streamline angle is about $45^{\circ}$ for a zero magnetic field and about $20^{\circ}$ for a $10 \mathrm{mT}$ magnetic field. Magnetic force from azimuthal self-magnetic field was also taken into account. It was found that the self-magnetic field does not affect either plasma jet shape, density, velocity and the current distribution for arc currents less than $200 \mathrm{~A}$. On the other hand the mass and the current flow are compressed in a high current vacuum arc that indicates significance of the self-magnetic field. While studying the geometry effect in combination with an axial magnetic field it was found that the near anode region plays an important role. For instance in the case of a ring anode vacuum arc, the plasma voltage and anode voltage contribute substantially to the total arc voltage. Arc voltage increase with imposition of a magnetic field and decreases with the anode length in agreement with experiment. Multiply charged ion transport in the free boundary vacuum arc plasma jet was studied. ${ }^{27,28}$ It was also found that the differently charged ions of the same element have approximately the same velocity. Keidar et al ${ }^{27,28}$ found that the charge state distribution is not non-uniform along the plasma jet due to the separation of different charge species. Due to the existence of an electric field in the quasi-neutral plasma the different charged ion species can be separated according to this model. It was found that the mean charge state distribution is strongly non-uniform with a tendency for a highly charged species to appear near the plasma jet boundary region. This prediction was confirmed by experiment. ${ }^{28}$

Above mentioned models however relayed on the assumption that plasma expansion is isothermal. Recent study of non-steady vacuum arc suggests that electron temperature may increase up to $10^{2} \mathrm{eV}$ at current of order $1 \mathrm{kA}$ due to current constriction $^{29}$. It was proposed that this effect may explain experimentally observed mean charge state increase with arc current ${ }^{30}$. However, one can ask whether such effect can take place in the steady state vacuum arc. In fact recent complementary analysis of the $2 \mathrm{D}$ energy balance indicated that even in the high-current vacuum arc the temperature changes are marginal. ${ }^{31}$ 
Plasma jet originates from the cathode spot having an effective radius $R_{o}$ (Fig. 2). According to the vacuum arc cathode jet model the plasma has density of about $10^{20}-10^{21} \mathrm{~m}^{-3}$ in the interelectrode gap at about $1 \mathrm{~mm}$ from the cathode plane ${ }^{17}$. In such a density range the influence of inelastic collisions (ionization, recombination, etc.) on the plasma density can be neglected. The plasma jet has a free radial boundary whose position will be determined as part of a self-consistent solution. The plasma jet model is based on the following assumptions and conditions: (1) the vacuum arc plasma is fully ionized; (2) during the expansion, the temperature of each species remains constant; (3) the mean free path for elastic electron-ion collisions is much smaller than the characteristic plasma jet radius; (4) the ions and electrons are ideal gases with partial pressures $\mathrm{P}_{\alpha}=\mathrm{kT}_{\alpha} \mathrm{N}_{\alpha}$, where $\alpha=\mathrm{e}, \mathrm{i}$, and k is Boltzmann's constant; (5) the electrons are magnetized and the ions are unmagnetized, i.e., $\rho_{\mathrm{I}}>\mathrm{R}_{\mathrm{o}}>>\rho_{\mathrm{e}}$, where $\rho_{\mathrm{e}}, \rho_{\mathrm{i}}$ are the electron and ion Larmor radii, respectively; (6) the plasma is quasi-neutral;

Briefly, the model is based on two-dimensional, axisymmetric, two-fluid magnetohydrodynamics, and includes mass and momentum conservation for both the ions and electrons. ${ }^{22-26}$. The following system of equations completely describes the plasma flow and current distribution:

$$
\begin{aligned}
& m_{\mathrm{i}}\left(\mathbf{V}_{\mathrm{i}} \cdot \nabla\right) \mathbf{V}_{\mathrm{i}}=-k\left(Z_{\mathrm{i}} T_{\mathrm{e}}+T_{\mathrm{i}}\right) \cdot \nabla \ln (n)+\mathbf{j} \times \mathbf{B} / n \\
& \mathbf{j}=\sigma\left\{\mathbf{E}+\left(k T_{\mathrm{e}} / e\right) \cdot \nabla \ln (n)-\mathbf{j} \times \mathbf{B} /(e n)+\left(\mathbf{V}_{\mathrm{i}} \times \mathbf{B}\right)\right\} \\
& \nabla \cdot\left(\mathbf{V}_{\mathrm{i}} n\right)=0 \\
& \nabla \cdot \mathbf{j}=0
\end{aligned}
$$

where $m_{\mathrm{i}}$ is the ion mass, $\mathbf{V}_{\mathrm{i}}$ is the ion velocity, $T_{\mathrm{e}}$ and $T_{\mathrm{i}}$ are electron and ion temperatures, $n$ is the plasma density, $\mathbf{E}$ is the electric field, $\mathbf{j}$ is the current density.

There are three boundaries where conditions must be specified: the cathode side, the anode side and the free lateral boundary. On the cathode side, we choose for the starting plane of the present $2 \mathrm{D}$ model some distance above the cathode spot where the results of the more detailed 1D near-cathode model discussed in [32] and the present 2D model are both valid. This is the point where the quasi-neutral plasma begins to freely expand above the cathode region where the plasma is generated. The previous $1 \mathrm{D}$ calculations further indicate that this plasma density corresponds to ion and electron temperatures of about $T_{i}=0.5 \mathrm{eV}$ and $T_{e}=2 \mathrm{eV}$, respectively and are independent of the spot current. The ion temperature is a measurement of the random thermal velocity that is superimposed on the much larger directed velocity. The initial jet radius $R_{o}$ is computed from $R_{o}=\left\{f_{i} I_{o} /\left(\pi Z_{i} e n_{o} V_{z o}\right)\right\}^{0.5}$, where $f_{i}$ is the ion current fraction $\left(f_{i}=0.1\right), I_{o}$ is the current per single spot, $V_{z o}=$ is the initial axial ion velocity, and $Z_{i}$ is the ion mean charge. The region between the cathode surface and the starting plane in the present model includes the total potential cathode fall. This is the net voltage drop between the contact surface and the beginning of the freely expanding plasma region. When the total arc voltage is calculated in the present model, the cathode fall value is added to the plasma voltage. We define the free plasma boundary as the surface where the plasma jet velocity has only a tangential component, i.e., the normal component $V_{n}$ is zero. The plasma density at the free boundary of the plasma jet is $n=0$, and normal current density is $j_{n}=0[12-26]$.

\section{Vacuum arc thruster plasma plume in a magnetic field}

In this section we present results of the calculation of the plasma plume exhaust from the vacuum arc thruster based on the plasma flow model described above. Specifically an effect of the magnetic field was studied. The considered geometry is shown in Fig. 2. Since we are interested in plasma plume expansion at large distances from the cathode surface $($ several $\mathrm{cm}$ ) it is necessary to take into account magnetic field variation in the axial direction (far from the magnetic coil):

$$
\frac{B(z)}{B_{o}}=\frac{1}{\left[1+\left(\frac{Z}{R_{c}}\right)^{2}\right]^{3 / 2}}
$$


where $B_{o}$ is the magnetic field the cathode and $R_{c}$ is the coil radius. Magnetic field distribution in the axial direction is shown in Fig. 3

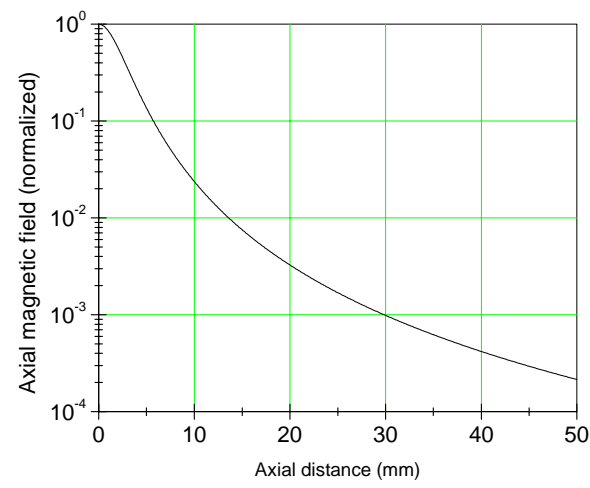

Figure 3. Magnetic field distribution (axial component) in axial direction.

The calculated plasma density distribution in the plasma plume exhaust from the MVAT is shown in Fig. 4. One can see that the magnetic field generally reduces plasma expansion. However this effect takes place mostly near the plasma generation region (where magnetic field is strong) and decreases in the axial direction. In order to enhance the focusing effect of the axial magnetic field the cathode should be placed inside the solenoid. However in this case arc voltage can increase due to decrease in conductivity across the magnetic field in the interelectrode gap. This effect will be considered in Sec. V.
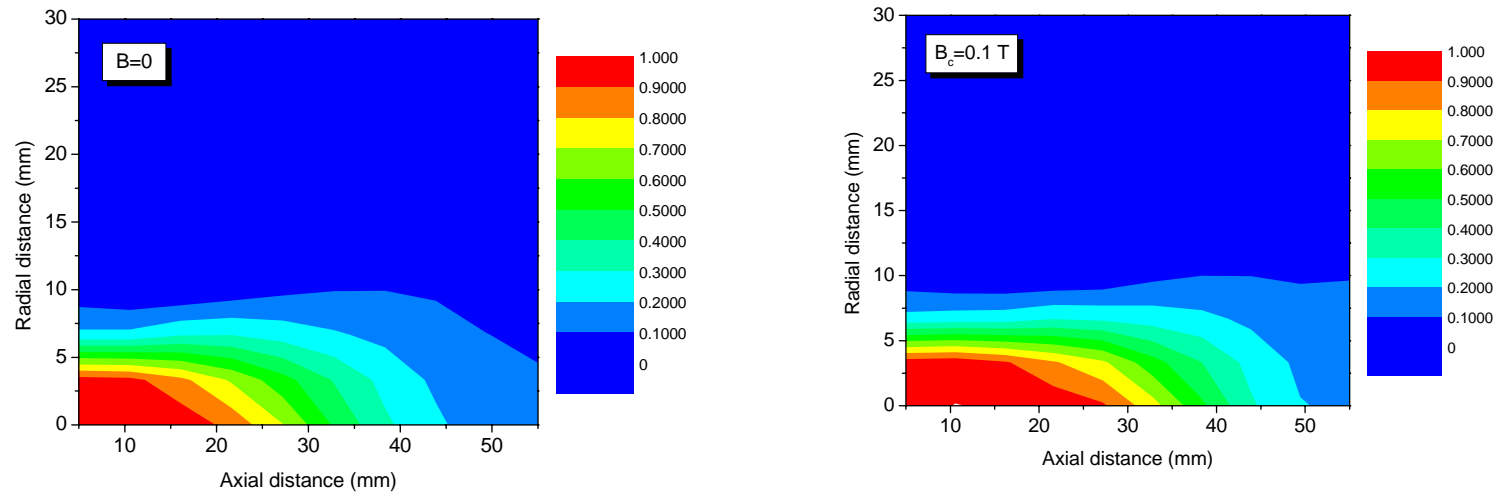

Figure 4. Comparison of the plasma density distribution with and without magnetic field. Arc current is 200 A. Magnetic field, $B_{0}$, is about $0.1 \mathrm{~T}$ at the cathode surface.

In order to validate the model we performed a detailed comparison between the model predictions and the experiment. The available experimental data contain radial and axial distribution of the ion current density (details can be found in a parallel pape ${ }^{21}$ ). The ion current density radial distribution is shown in Fig. 5 for several axial positions. Two cases are compared with no magnetic field and with an axial magnetic field $\left(B_{o}=0.5 \mathrm{~T}\right)$. Plasma spreads significantly at large distances from the thruster and plasma plume develops a half angle of about $45^{\circ}$. One can see that the magnetic field leads to plasma focusing and as a result the ion current density along the axis $(r=0)$ is high. It can be seen that the experimental and calculated distributions generally agree well and show the similar trends. 

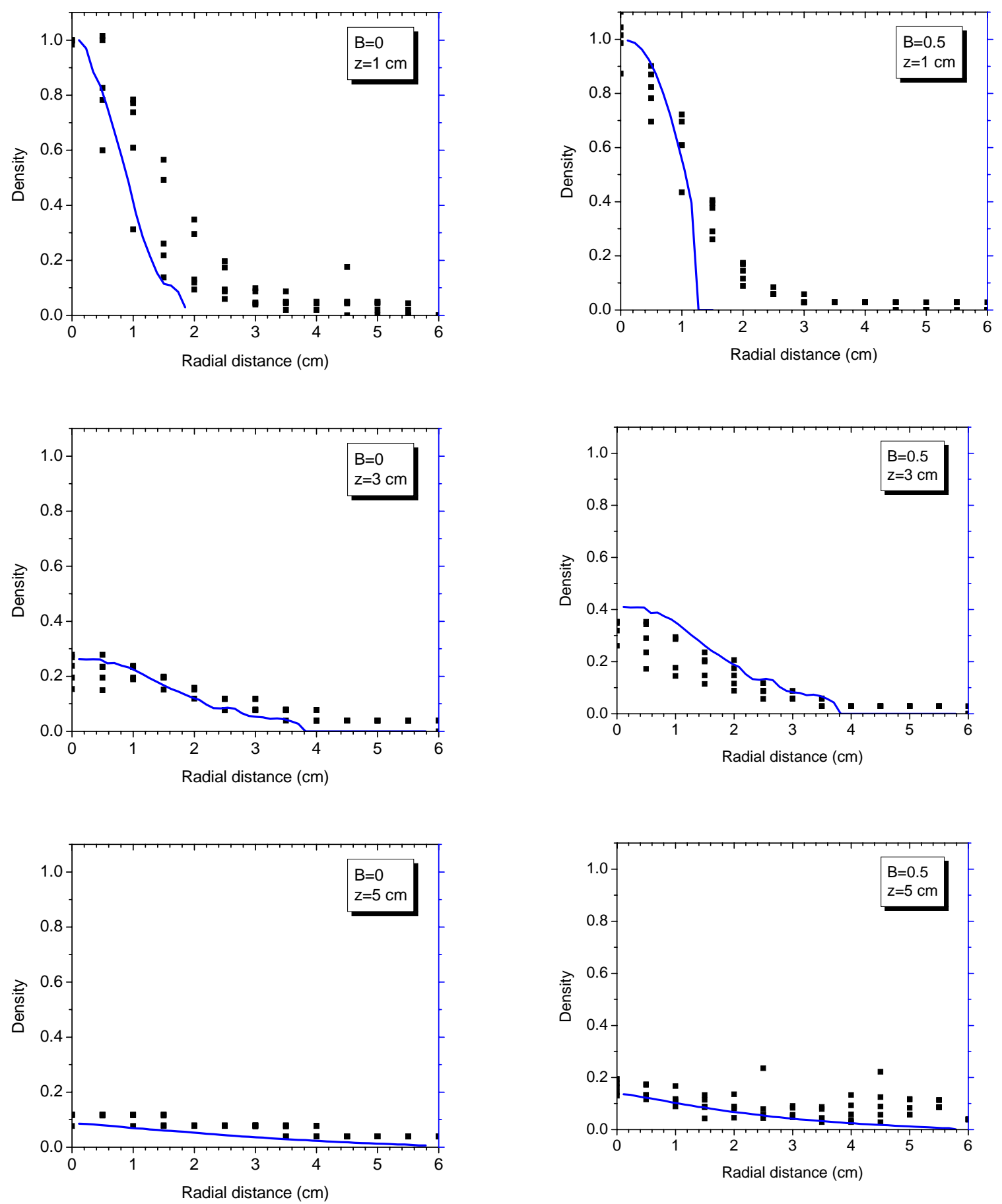

Figure 5. Plasma density distribution with and without axial magnetic field. Comparison with experiment. (experimental data: solid squares). Magnetic field near the cathode, $B_{0}=0.5 \mathrm{~T}$ 


\section{Arc voltage}

It was suggested above that the possible way to enhance effect of the magnetic field is to place cathode inside of the solenoid. However in this case arc voltage can increase due to decrease in conductivity across the magnetic field in the interelectrode gap. Similarly due to cathode erosion and therefore cathode recession after long time operation of the VAT the cathode surface will be placed inside the anode tube. Therefore it is important to understand what effect it may have on the arc voltage.

The potential distribution in the interelectrode gap can be calculated from current conservation equation:

$$
\frac{\partial j_{r}}{\partial r}+\frac{\partial j_{z}}{\partial z}+\frac{j_{r}}{r}=0
$$

where $j_{r}$ and $j_{z}$ are radial and axial components of the current density respectively and are determined from Eq. 2. The numerical analysis is similar to that developed previously. ${ }^{22-26}$ We use the implicit two-layer method to solve the system of Equations (1)-(4). These equations are approximated by a two-layer, six-point scheme. An iterative procedure for finding the plasma density, velocity and potential distribution self-consistently is employed. The equation for potential (Eq. 5) is solved numerically by iteration using the successive overrelaxation procedure.

The calculated potential distribution in the interelectrode gap is shown in Fig. 6 for $\mathrm{B}=0$ and arc current of about $200 \mathrm{~A}$.

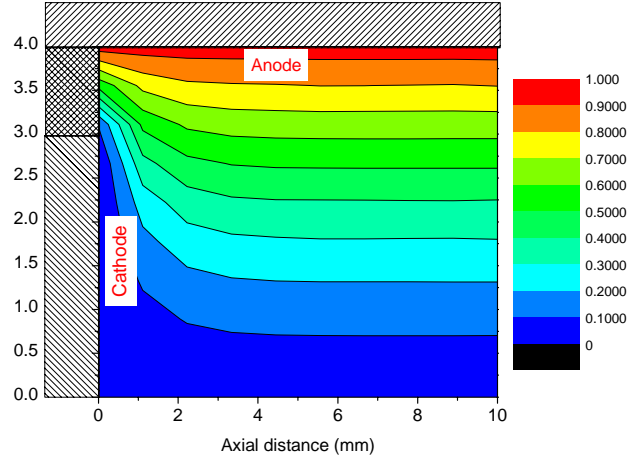

Figure 6. Potential distribution in the vacuum arc interelectrode gap of MVAT. $B=0$. The anode length is $10 \mathrm{~mm}$. Arc current is $200 \mathrm{~A}$. The cathode voltage drop is not included.

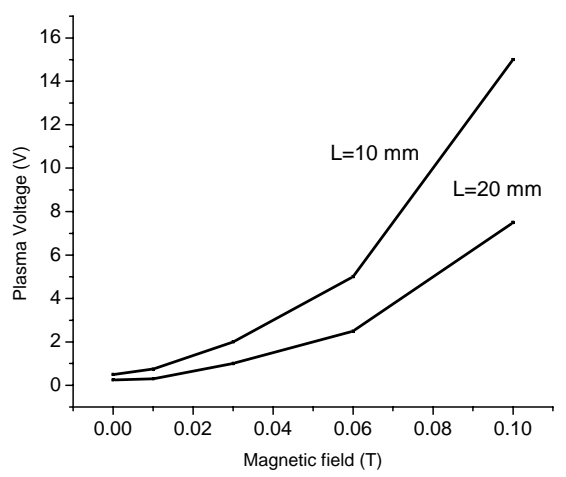

Figure 7. Vacuum arc voltage vs magnetic field with the anode length $L$ as a parameter. Arc current is $200 \mathrm{~A}$. The cathode voltage drop is not included.

Application of the axial magnetic field leads to the arc voltage increase as shown in Fig. 7. It can be seen that longer anode (cathode inside the solenoid) leads to low arc voltage. On the other hand plasma losses to the wall increase. Therefore one can conclude that the optimization of the interelectrode gap geometry (mainly anode length) is possible in order to keep low arc voltage while providing strong plasma collimation. 


\section{Modeling of the ring electrode vacuum arc thruster}

Very effective feed mechanism is possible if cathode is the walled tube as shown in Fig. 8 (Ref. 33). However in this case the thrust vector is not directed along the axis of symmetry and thrust is generated by creation pressure gradient from the tube, i. e VAT operate as an electrothermal thruster. In order to provide efficient thrust in this geometry and to preserve high ion velocity (specific impulse) a magnetic field can be used.

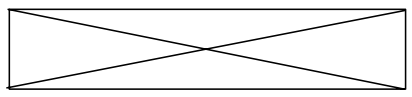

Insulator

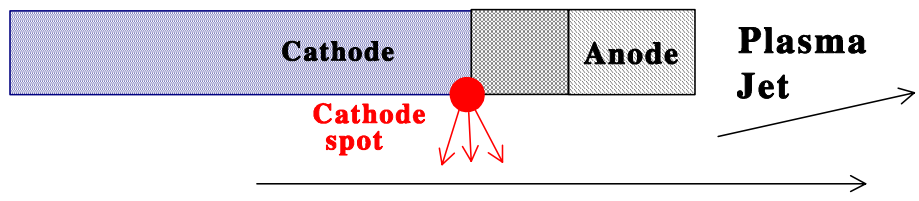

B
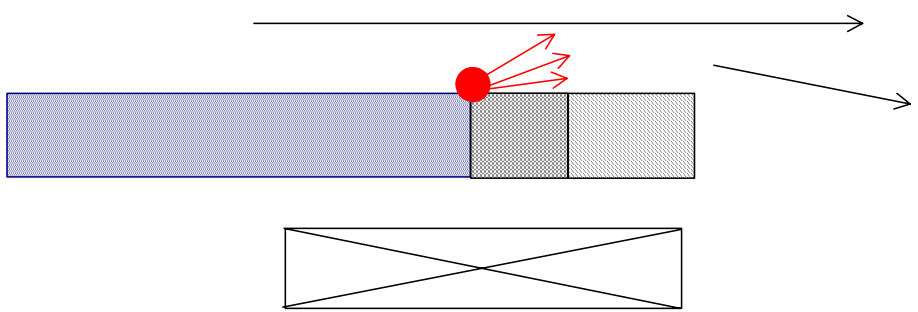

Figure 8. Feed mechanism and schematics of plasma collimation

In this section we present analysis of the plasma flow inside the tube with an aim to explain experimentally observed non-monotonic behavior of the ion current density with an axial magnetic field. The calculations are based on the model described in the Sec. III.

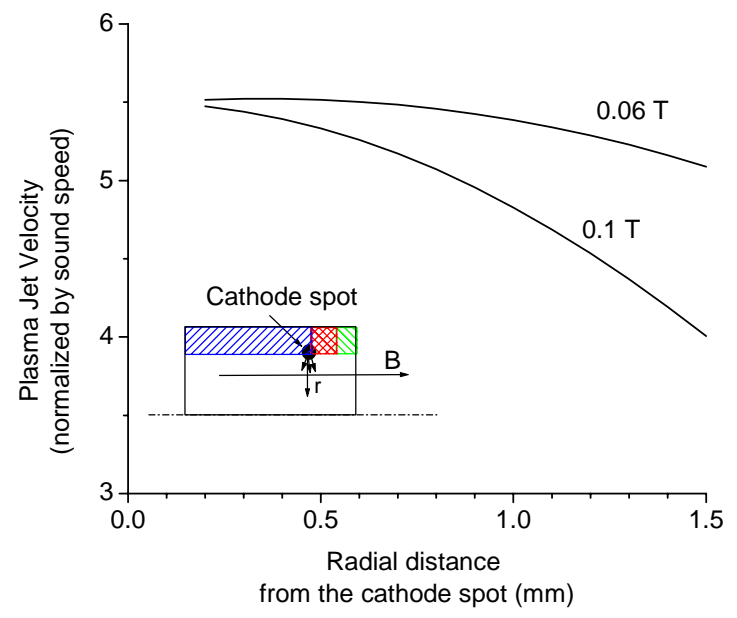

Figure 9. Calculated plasma jet velocity with a magnetic field as a parameter 
The plasma jet expansion from the cathode spot across the magnetic field was calculated. The velocity distribution is shown in Fig. 9 with magnetic field as a parameter. One can see that plasma is decelerated in the course of flow across the magnetic field. It happens due to the electron magnetization and preservation of the plasma quasi-neutrality condition.

Calculated ion density distribution inside the tube (dielectric and anode) is shown in Fig. 10. It can be seen that an axial magnetic field significantly affect the plasma flow. One can see that plasma is significantly collimated due to the magnetic field effect.
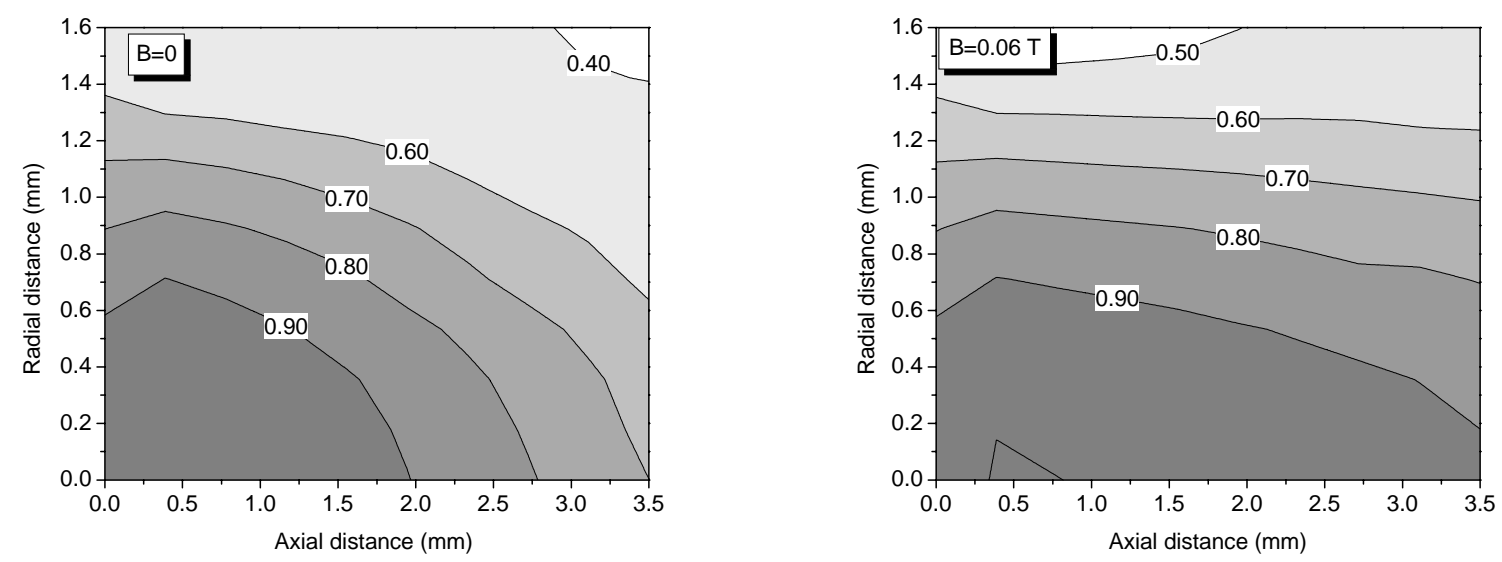

Figure 10. Ion current density distribution inside the tune between the cathode plane and the exit.

However these calculations were performed for the same starting conditions, i.e. effect of the magnetic field on the starting conditions was not included. It was shown (Fig. 9) that magnetic field affects significantly plasma velocity if the plasma flows across the magnetic field. Therefore the change of the starting conditions (plasma velocity) should be taken into account.

Two limited cases are possible with plasma jet propagation across the magnetic field in the cathode spot vicinity (and therefore decrease of the plasma velocity) and plasma jet propagation along the magnetic field as shown schematically in Fig. 8. The calculated ion current density at the thruster exit plane for these two cases is shown in Fig.11. One can see that ion current density increases with magnetic field increase due to plasma collimation effect; reaches some maximum and then decreases due to plasma velocity decrease. On the other hand ion current density in the plasma jet propagated along the magnetic field experiences monotonic growth with magnetic field and then saturated at high magnetic field due to collimation effect. For comparison the experimental data are shown. It can be seen that the model prediction about non-monotonic behavior of the exhaust ion current density with magnetic field is in agreement with experimental trend. 


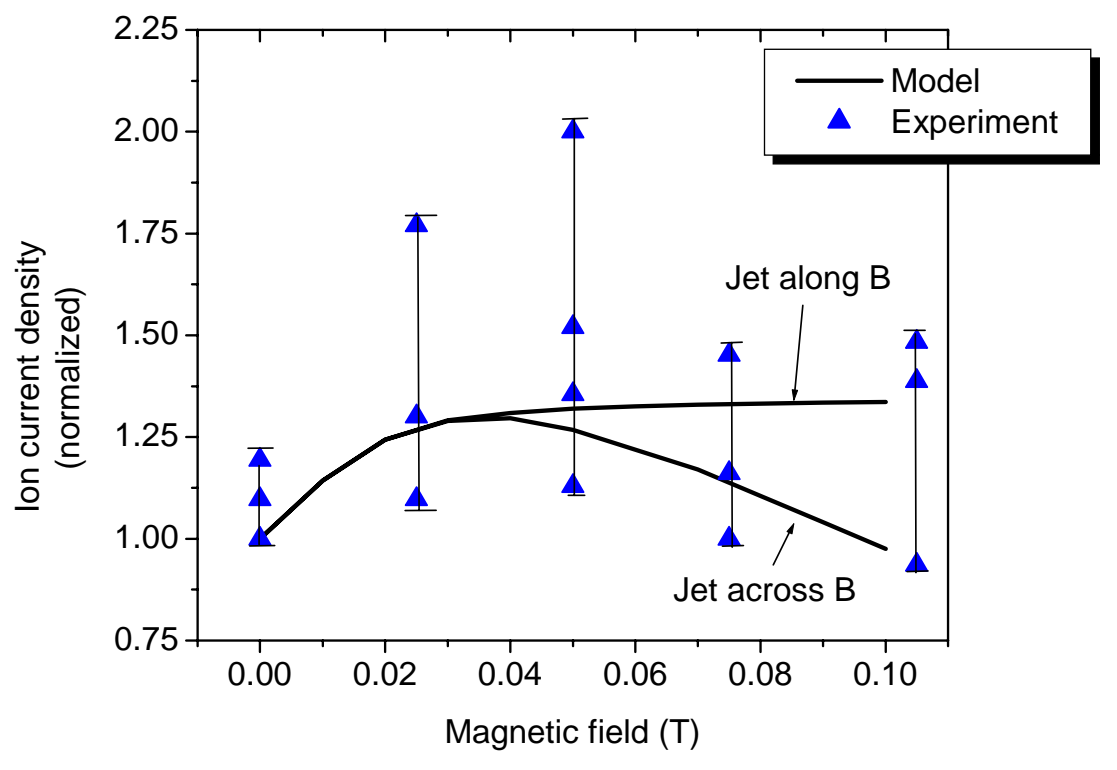

Figure 11. Ion current density (exit plane) dependence on the magnetic field inside the tube. Comparison of the model predictions and experiment.

\section{Concluding Remarks}

In this paper the hydrodynamic model of the vacuum arc thruster and its plume is developed. An effect of the magnetic field on the plume expansion and thruster operational conditions is considered. In particular it was found that magnetic field significantly reduces plume expansion while on the other hand leads to arc voltage growth dependent on thruster geometry. Two specific examples are investigated namely magnetically enhanced co-axial vacuum arc thruster (MVAT) and vacuum arc thruster with ring electrodes (RVAT). Model predictions were validated against experimental data and generally good agreement was found. In the case of RVAT it was found that magnetic field leads to plasma jet deceleration. This effect explains non-monotonic dependence of the ion current density with an axial magnetic field observed experimentally.

\section{Acknowledgments}

This work was funded by NASA SBIR Contract No NNC04CA19C.

\section{References}

${ }^{1}$ B. E. Djakov and R. Holmes, J. Phys. D: Appl. Phys., 7, p. 569, 1974.

${ }^{2}$ G.A. Lyubimov and V.I. Rakhovsky, Sov. Phys. Usp., 21, p.693, 1978.

${ }^{3}$ V.I. Rakhovsky, IEEE Trans. Plasma Sci., 15, p.481, 1987.

${ }^{4}$ J. Kutzner and H.C. Miller, J. Phys. D: Appl. Phys., 25 1992, pp. 686-693.

${ }^{5}$ A. A. Plyutto, V. M. Ryzhkov, and A. T. Kapin, 1965, Sov. Phys. JETP, 20, 328.

${ }^{6}$ W.D. Davis and H.C. Miller, J. Appl. Phys., 40, p. 2212, 1969.

${ }^{7}$ Vacuum Arcs-Theory and Applications, edited by J.M. Lafferty (Wiley, New York 1980). 
${ }^{8}$ Vacuum Arc Science and Technology, edited by R.L. Boxman, P.J. Martin and D. M. Sanders (Noyes, New York, 1995).

${ }^{9}$ I.I. Aksenov, V.G. Padalka, and V.M. Khoroshikh, , Sov. J. Plasma Phys., 5(3), 1979, 341-344.

${ }^{10}$ Y. Cohen, R.L. Boxman and S. Goldsmith, Angular distribution of ion current emerging from an aperture anode in a vacuum arc, IEEE Trans. Plasma Sci., 17 (5) pp. 713-716, OCT 1989.

${ }^{11}$ I.G. Brown, Rev. Sci. Instr. 65, 30611994.

${ }^{12}$ E.M. Oks, I.G. Brown, M.R. Dickinson, R.A. MacGill, H. Emig, P. Spadtke and B.H. Wolf, Appl. Phys. Lett, 67, 200, 1995.

${ }^{13}$ G. Yushkov, A. Anders, E. Oks and I. G. Brown, J. Appl. Phys., 88, p. 5618, 2000.

${ }^{14}$ G.A. Lyubimov, Sov. Phys. Tech. Phys., 22, 173, 1977.

${ }^{15}$ C. Wieckert, Phys. Fluid, 30 (6) 1810, 1987.

${ }^{16}$ I. I. Beilis, M. P. Zektser, G. A. Lyubimov, Sov. Phys. Tech. Phys., 33, 1988, pp. 1132-1137.

${ }^{17}$ I.I. Beilis and M. P. Zektser, High Tempr., vol. 29, 1991, pp. 501-504.

${ }^{18}$ J.Schein, A. Anders, R. Binder, M. Krishnan, J. E. Polk, N. Qi and J. Ziemer, Review of Scientific Instruments, vol. 72, no 3. Feb. 2002

${ }^{19}$ Schein, J., Krishnan, M., Ziemer, J., Polk, J, "Adding a "Throttle" to a Clustered Vacuum Arc Thruster" AIAA paper 2002-5716, Nanotech, 2002

${ }^{20}$ Schein, J., Gerhan, A., Rysanek, F., Krishnan, M. "Vacuum Arc Thruster for Cubesat Propulsion" IEPC-0276 , $28^{\text {th }}$ IEPC, 2003

${ }^{21}$ M.Au, J.Schein, A. Gerhan, K. Wilson, B. Tang, M. Krishnan, AIAA Paper 2004-3618, July 2004

${ }^{22}$ M. Keidar, I.I. Beilis, RL. Boxman and S. Goldsmith, 2-D Expansion of the low-density interelectrode vacuum arc plasma jet in an axial magnetic field, J. Phys. D: Appl. Phys., 29, 1996, pp. 1973-1983.

${ }^{23}$ I. Beilis, M. Keidar, R. L. Boxman, and S. Goldsmith. Theoretical study of plasma jet expansion in a magnetic field in a disc anode vacuum arc. J. Appl. Phys., 83 (2), 1997, pp. 709-717.

${ }^{24}$ M. Keidar, I. Beilis, R. L. Boxman, S. Goldsmith. Voltage of the vacuum arc with a ring anode in an axial magnetic field. IEEE Trans. Plasma Sci., 25, 1997, pp. 580-585.

${ }^{25}$ I. I. Beilis, M. Keidar, R. L. Boxman and S. Goldsmith, The model of plasma expansion and current flow in a vacuum arc with a small anode. IEEE Trans. Plasma Sci., 27, 1999, pp. 872-876.

${ }^{26}$ M. Keidar and M.B. Schuman, IEEE Trans. Plasma Sci., Vol. 29, No. 5, pp. 684-689, Oct. 2001

${ }^{27}$ M. Keidar, I. I. Beilis and I. G. Brown, J. Appl. Phys., 84 1998, pp. 5956-5960.

${ }^{28}$ M. Keidar, I.G. Brown and I.I. Beilis, Review of Scientific Instrument, 71, 2000, pp. 698-700.

${ }^{29}$ E.A. Zverev and I.A. Krinberg, Tech. Phys. Lett., 24, 1998, pp. 728-730.

${ }^{30}$ Oks E, Anders A, Brown I. G, Dickinson M R, and MacGill R A, 1996 IEEE Tans. Plasma Sci., 24 pp. 11741183 .

${ }^{31}$ I.I. Beilis and M. Keidar, Proc. XXth Inter. Sympos. Disch. Elect. Insul. Vacuum, 2002 (Tours, France), pp. 535538.

${ }^{32}$ I.I. Beilis in Handbook of vacuum arc science and technology, edited by R.L. Boxman, P.J. Martin and D. M. Sanders (Noyes, New York, 1995).

${ }^{33}$ Wilson, K., Schein, J., Gerhan, A., Au, M., Krishnan, M. AIAA Paper AIAA 2004-3617, July 2004. 